\title{
Variación entre años de las infracomunidades de parásitos metazoos de la corvina Cilus gilberti (Pisces: Sciaenidae) en Chile
}

\author{
Between-years variation in infracommunities of metazoan parasites of the croaker \\ Cilus gilberti (Pisces: Sciaenidae) from Chile
}

FÉLIX GARCÍAS ${ }^{1}$, RICARDO MENDOZA² \& MARIO GEORGE-NASCIMENTO ${ }^{1}$

${ }^{1}$ Facultad de Ciencias, Universidad Católica de la Santísima Concepción, Casilla 297, Concepción, Chile; e-mail: mgeorgen@ucsc.cl

${ }^{2}$ Biotecmar Servicios, Universidad Católica de la Santísima Concepción, Colón 2766, Talcahuano, Chile

\begin{abstract}
RESUMEN
Se comparan la composición, riqueza, diversidad, abundancia y dominancia en las infracomunidades de parásitos metazoos de 86 ejemplares de corvina Cilus gilberti (Abbott 1889), recolectados en dos muestras tomadas con 15 años de diferencia en la zona de Talcahuano, VIII región, Chile. Se pretende averiguar si estas características comunitarias se modifican o no sustantivamente entre años. Se recolectaron un total de 26 taxa, a los que se les determinó la prevalencia, aunque sólo a 22 se les determinó la abundancia. El $69 \%$ de los 26 taxa ocurría en ambos años, sin embargo, al comparar la composición infracomunitaria entre años, sólo se consideró a aquellos 13 taxa que estaban en al menos 10 corvinas del total de 86 examinadas, y a hospedadores de similar longitud corporal. Hay variaciones significativas entre años en la composición de las infracomunidades de parásitos pero no en la riqueza, abundancia, diversidad y dominancia. Aunque los antecedentes son escasos, la observada relativamente alta variabilidad composicional y baja variabilidad agregada es similar a lo encontrado en infracomunidades de parásitos, tanto de peces como de mamíferos. Se discute la medida en que estos resultados representan compensación o estasis comunitaria, entre cuyas causas podrían estar las interacciones biológicas.
\end{abstract}

Palabras clave: infracomunidades de parásitos, composición, diversidad, comparación entre años, Cilus gilberti, Chile.

\section{ABSTRACT}

The composition, richness, diversity, abundance and dominance in infracommunities of metazoan parasites found in 86 croaker Cilus gilberti (Abbott 1889) are compared between two samples taken off Talcahuano, Chile, with 15 years of difference. All hosts harboured parasites, and 26 taxa were collected. The prevalence of all taxa, and the abundance of 22 taxa were recorded. Most $(69 \%)$ taxa were found in both years. However, to compare between years, we just considered those 13 taxa occurring in at least 10 host individuals. Significant variations in composition but not in richness, abundance, diversity and dominance were found when infracommunities collected from croaker of similar body length were compared between years. Despite the fact that there are few antecedents on the subject, these results are similar to those reported in infracommunities of metazoan parasites of fishes and mammals in that there is high compositional variability and low aggregate variability. The extent to which this can be interpreted as indicating community compensation or stasis is discussed, considering that biological interactions could also be responsible for the observed pattern.

Key words: infracommunities, composition, diversity, between-year comparisons, Cilus gilberti, Chile.

\section{INTRODUCCIÓN}

Gran parte de los estudios en las variaciones temporales de la composición comunitaria, diversidad, riqueza de especies etc, se han realizado en el marco de la teorías de sucesión, o bien de descripciones de secuencias fenológicas (ver Pimm 1991, Ricklefs \& Schluter 1993, Hubbell 2001, Jaksic 2001). Los ecólogos de comunidades de plancton están entre los que han realizado los registros comunitarios de composición y di- versidad de más largo plazo, en tiempo ecológico (McGowan \& Walker 1993), aunque aún no han efectuado análisis conjuntos de la variabilidad en composición y en diversidad (o riqueza de especies). En las comunidades de parásitos, como en otras, los estudios se realizan mayoritariamente sólo en un momento del tiempo, en un lugar de estudio, o en una especie de hospedador (Esch et al. 1990a, 1990b, Sousa 1994, Poulin \& Morand 2000). 
En el estudio de las comunidades de parásitos se reconocen básicamente tres niveles jerárqui$\cos$. Las infracomunidades, que incluyen a todos los individuos de las distintas especies de parásitos dentro de un individuo hospedador. La comunidad componente, que está formada por todas las infracomunidades dentro de una población o especie de hospedador, y finalmente, la comunidad compuesta, que incluye a todas las comunidades de parásitos en los distintos hospedadores dentro de un ecosistema (Holmes \& Price 1986). Este estudio se enmarca en el nivel infracomunitario. Los patrones descritos en composición y riqueza de especies parásitas han sido interpretados en forma diversa, entre los que se cuentan los que se relacionan a la dieta, a la edad, origen geográfico de los hospedadores, así como a interacciones biológicas, o a simples diferencias demográficas entre las especies de parásitos (Poulin 1997, Vickery \& Poulin 1998, Poulin \& Morand 2000).

Los pocos estudios acerca de la variabilidad temporal de las infracomunidades de parásitos han evaluado la similitud entre años consecutivos en composición, diversidad y otros descriptores infracomunitarios (Holmes 1990, Kennedy 1993, Thoney 1993, Balboa \& George-Nascimento 1998). Recientemente, se observaron variaciones no significativas de la diversidad, equitabilidad y riqueza de especies en el tiempo, entre estaciones de pastoreo, estado de desarrollo, etc., en ensambles de nematodos que habitan en el tracto digestivo de ovejas. Sin embargo, hubo cambios menores en composición, indicando una gran estabilidad tanto en el tiempo como a través de diversas características del hospedador (Gruner et al. 1998)

En este trabajo se investiga si la composición, riqueza, diversidad, abundancia total y dominancia en las infracomunidades de parásitos de la corvina Cilus gilberti (Abbott 1889) (Perciformes: Sciaenidae) se modifican o no sustantivamente entre muestras tomadas con 15 años de diferencia en una misma localidad. La gran diferencia en tiempo entre las muestras mejora notoriamente la posibilidad de que se esté haciendo observaciones sobre generaciones distintas de parásitos y de hospedadores. Además, tenemos la certeza de no sólo no estar haciendo observaciones sobre los mismos sitios, sino sobre distintos individuos parásitos, ya que el muestreo destructivo de las infracomunidades lo impide. En trabajos anteriores se ha recomendado hacer estas comparaciones entre hospedadores de similar edad, ya que no es inusual que hayan cambios ontogenéticos en la ecología del hospedador (George-Nascimento \& Iriarte 1989, Balboa \& George-Nascimento 1998). En términos generales, la variación ontogenética de la dieta del hospedador juega un rol importante en el reclutamiento de los endoparásitos ya que puede explicar patrones de abundancia de algunos taxa en función de la longitud corporal del hospedador. Por esto, en este estudio enfatizamos en las comparaciones entre infracomunidades de edad similar (= hospedadores de similar tamaño). Cabe destacar que, en general, cada especie de hospedador posee una fauna parasitaria propia ya que la mayoría de los parásitos son específicos. Por esto, las diferencias en composición infracomunitaria dentro de una especie de hospedador, son en términos de abundancia relativa, y sobre un mismo conjunto de especies de parásitos (la comunidad componente).

La corvina Cilus gilberti (Perciformes: Sciaenidae) es de hábitos neríticos y ocupa fondos arenosos o fangosos, donde se alimenta principalmente de pequeños crustáceos (misidáceos y eufáusidos) y pequeños peces clupeiformes. Posee una dieta que se modifica ontogenéticamente (Chong et al. 2000). En Chile es un recurso pesquero en explotación desde Arica a Chiloé (Kong \& Valdés 1990). Hasta antes de 1985 se consideraba a Cilus montti y a Sciaena gilberti como dos especies diferentes. Sin embargo, Oyarzún et. al. (1985) definen el status taxonómico de la corvina ubicando a estas dos especies en una, por lo que finalmente se propone a Cilus gilberti como única especie. En Chile, Mendoza (1983) registró la fauna de parásitos metazoos de 57 corvinas (Cilus gilberti) recolectadas en la localidad de Talcahuano, por otro lado Bolaños \& Salas (1982) registraron a Villarrealina peruanus en el tracto digestivo de Sciaena gilberti, y Oliva \& Durán (1982) registraron al copépodo Lernanthropus pacificus en la cavidad branquial de la misma especie de hospedador.

\section{MATERIALES Y MÉTODOS}

Las infracomunidades de parásitos fueron obtenidas de 57 y 29 corvinas muestreadas en julio de 1983 y abril de 1998 , respectivamente, en la zona de pesca vecina al puerto de Talcahuano $\left(36^{\circ} 41^{\text {, }}\right.$ $S, 73^{\circ} 06^{\prime} \mathrm{O}$ ), Chile. Los datos de la muestra de 1983 fueron obtenidos por Mendoza (1983) quien no evaluó la abundancia de Bucephalidae gen. sp., Plagioporus sp., Cryptogonimidae gen. sp. y Philometridae gen. sp. Por esto, se excluyen estos 4 taxa cuando se examina la correlación de las abundancias de cada taxón entre años. Ejemplares de cada taxón hallado en la muestra de 1983 estuvieron disponibles para comparación con los de la muestra de 1998. 
Los hospedadores fueron preservados a $-20^{\circ} \mathrm{C}$ hasta el censo de cada infracomunidad, mediante los métodos descritos en George-Nascimento \& Iriarte (1989). Una vez descongelados, se procedió a la obtención de la longitud total $(\mathrm{cm})$ de cada hospedador. La longitud corporal de las corvinas fue comparada entre años mediante una prueba de Kolmogorov-Smirnov para dos muestras (Zar 1984).

Se supone que la longitud total de los hospedadores representa la edad de las corvinas (= de las infracomunidades). La longitud total de las 57 corvinas recolectadas en 1983 fluctuó entre 51,6 y $76,5 \mathrm{~cm}$, y la de las 29 recolectadas en 1998 fluctuó entre 32,5 y $75,0 \mathrm{~cm}$. La muestra tomada en 1998 abarcó un mayor intervalo de longitudes, ya que había una mayor proporción de ejemplares pequeños en dicha muestra (Dmáx. $=0,41,11$ intervalos, $\left.\mathrm{c}^{2}=13,1, \mathrm{~g} .1 .=2, \mathrm{P}=0,001\right)$. Por ello, y para evaluar el potencial efecto de las diferencias en longitud total de las corvinas de ambas muestras, se usaron dos aproximaciones para el análisis de la variación entre años de la composición y otras características infracomunitarias. En una se consideró a todas las infracomunidades muestreadas $(n=86)$; y en la otra se consideró sólo a las de corvinas de similar longitud total (mayores a $41 \mathrm{~cm}, \mathrm{n}=77$ ).

La determinación taxonómica de los peces fue realizada según los criterios de Oyarzún et al.

TABLA 1

Prevalencia (Pre, \%), número de individuos recolectados (Ind) y abundancia (Abu) de 26 taxa parasitarios encontrados en 86 ejemplares de Cilus gilberti muestreados en 1983 y 1998 en

Talcahuano, Chile

Prevalence (Pre, \%) number of collected individuals (ind) and abundance (abu) of 26 parasite taxa found in 86 Cilus gilberti sampled in 1983 and 1998 at Talcahuano, Chile

\begin{tabular}{|c|c|c|c|c|c|c|c|}
\hline \multirow[b]{2}{*}{ Taxón } & & \multicolumn{3}{|c|}{$\begin{array}{c}\text { Año } 1983 \\
(\mathrm{n}=57)\end{array}$} & \multicolumn{3}{|c|}{$\begin{array}{c}\text { Año } 1998 \\
(\mathrm{n}=29)\end{array}$} \\
\hline & & Pre & Ind & $\mathrm{Abu}$ & Pre & Ind & Abu \\
\hline \multicolumn{8}{|l|}{ Endoparásitos larvales } \\
\hline Corynosoma sp. & $\mathrm{AC}$ & 92,9 & 927 & 16,3 & 58,6 & 91 & 3,1 \\
\hline Nybelinia sp. & $\mathrm{CE}$ & 7,0 & 7 & 0,1 & 10,4 & 5 & 0,2 \\
\hline Diphyllobothridae gen. sp. & $\mathrm{CE}$ & 1,8 & 1 & $<0,1$ & 13,8 & 17 & 0,6 \\
\hline Scolex pleuronectis & $\mathrm{CE}$ & 0 & 0 & 0 & 10,3 & 15 & 0,5 \\
\hline Anisakis sp. & $\mathrm{NE}$ & 17,5 & 41 & 0,7 & 75,9 & 97 & 3,3 \\
\hline Contracaecum sp. & NE & 82,5 & 418 & 7,3 & 13,8 & 6 & 0,2 \\
\hline Hysterothylacium sp. & $\mathrm{NE}$ & 29,8 & 49 & 0,9 & 6,9 & 2 & 0,1 \\
\hline Pseudoterranova cattani & NE & 63,2 & 153 & 2,7 & 79,3 & 139 & 4,8 \\
\hline Proleptus sp. & NE & 1,8 & 1 & $<0,1$ & 0 & 0 & 0 \\
\hline Lacistorhynchus tenuis & $\mathrm{CE}$ & 40,4 & 50 & 0,9 & 0 & 0 & 0 \\
\hline \multicolumn{8}{|l|}{ Endoparásitos adultos } \\
\hline Rhadinorhynchidae gen. sp. & $\mathrm{AC}$ & 8,8 & 8 & 0,1 & 0 & 0 & 0 \\
\hline Bucephalidae gen. sp. & DI & 77,2 & - & - & 13,8 & 23 & 0,8 \\
\hline Villarrealina peruanus & $\mathrm{DI}$ & 38,6 & 40 & 0,7 & 100 & 1354 & 46,7 \\
\hline Plagioporus sp. & DI & 100 & - & - & 93,1 & 3066 & 105,7 \\
\hline Cryptogonimidae gen. sp. & DI & 100 & - & - & 62,1 & 283 & 9,8 \\
\hline Ascarophis sp. & $\mathrm{NE}$ & 0 & 0 & 0 & 41,4 & 31 & 1,1 \\
\hline Philometridae gen. sp. & $\mathrm{NE}$ & 35,1 & - & - & 17,2 & 8 & 0,3 \\
\hline Capillaria sp. & NE & 0 & 0 & 0 & 10,4 & 5 & 0,2 \\
\hline & Ectoparásitos & 0 & 0 & 0 & 3,5 & 1 & $<0,1$ \\
\hline Choricotyle sp. & MO & 82,5 & 190 & 3,3 & 37,9 & 26 & 0,9 \\
\hline Microcotylinae gen. sp. & MO & 28,1 & 26 & 0,5 & 72,4 & 86 & 3,0 \\
\hline Lepeophtheirus sp. & AR & 1,8 & 1 & $<0,1$ & 31,0 & 15 & 0,5 \\
\hline Lernanthropus pacificus & AR & 87,7 & 333 & 5,8 & 86,2 & 173 & 6,0 \\
\hline Neobrachiella sp1 & AR & 7,0 & 36 & 0,6 & 13,8 & 25 & 0,9 \\
\hline Neobrachiella sp2 & AR & 7,0 & 5 & 0,1 & 20,7 & 6 & 0,2 \\
\hline Caligus sp. & $\mathrm{AR}$ & 0 & 0 & 0 & 10,4 & 5 & 0,2 \\
\hline
\end{tabular}

$\mathrm{AC}=$ Acanthocephala, $\mathrm{CE}=$ Cestoda $, \mathrm{NE}=\mathrm{Nematoda}, \mathrm{DI}=$ Digenea, $\mathrm{MO}=$ Monogenea

$\mathrm{AR}=$ Arthropoda,$-=$ No determinado 
TABLA 2

Resultados de análisis de covarianza de la riqueza, abundancia total, diversidad, dominancia y composición de las infracomunidades de parásitos metazoos de la corvina, considerando a todas las corvinas (A) y aquellas de más de $41 \mathrm{~cm}$ (B), donde la longitud total del hospedador es la covariable, y el año de muestreo el factor. Se indican los valores de $\mathrm{F}$ y de probabilidad (P) de las pruebas de comparación de pendientes y de medias ajustadas por la covariable

Results of analyses of the covariance of richness, total abundance, diversity, dominance and composition of infracommunities of metazoan parasites of croaker $(A=$ all croakers and $B=$ croakers larger than $41 \mathrm{~cm})$. Total body length is the covariable and the year of sampling is the factor. F-ratios and probability values (P) after comparison of slopes and adjusted means are indicated

(A) Todas las corvinas

\begin{tabular}{|c|c|c|c|c|c|}
\hline & & & ación de per & & \\
\hline & Riqueza & Abundancia & Diversidad & Dominancia & Composición \\
\hline Valor de $F_{(1,82)}$ & 5,11 & 0,44 & 15,72 & 11,31 & 27,21 \\
\hline Valor de $\mathrm{P}$ & 0,03 & 0,51 & $<0,01$ & $<0,01$ & $<0,01$ \\
\hline & & Con & ón de media & adas & \\
\hline Valor de $F_{(1,83)}$ & - & 0,40 & - & - & - \\
\hline Valor de $\mathrm{P}$ & - & 0,53 & - & - & - \\
\hline (B) Corvinas de & $1 \mathrm{~cm}$ & & & & \\
\hline & & & ación de per & & \\
\hline Valor de $F_{(1.73)}$ & 0,40 & 1,41 & 2,05 & 0,81 & 5,24 \\
\hline Valor de $\mathrm{P}^{(1,}$ & 0,53 & 0,24 & 0,16 & 0,37 & 0,03 \\
\hline & & Con & ón de media & adas & \\
\hline Valor de $F_{(1.74)}$ & 0,26 & 1,34 & 0,00 & 0,02 & - \\
\hline Valor de $\mathrm{P}^{(1}$ & 0,61 & 0,25 & 0,97 & 0,88 & - \\
\hline
\end{tabular}

(1985) y Kong \& Valdés (1990), y la de los parásitos según Stuardo \& Fagetti (1961), Schmidt (1970), Cannon (1977), Deardorff \& Overstreet (1981), Bolaños \& Salas (1982), Oliva \& Durán (1982) y Villalba \& Fernández (1984). Los parásitos fueron fijados en formalina al $5 \%$.

La magnitud de las parasitosis en ambos años fue descrita mediante la prevalencia y la abundancia (Margolis et al. 1982). Se realizaron análisis de correlación de Spearman con el objetivo de indagar si los taxa que eran más prevalentes ( =26) y/o abundantes $(n=22)$ en un año lo eran también para el otro, lo que podría explicar en parte las potenciales variaciones encontradas.

Se calcularon los siguientes descriptores infracomunitarios: la riqueza infracomunitaria como el número de taxa presente en un individuo hospedador, la abundancia total como la suma de todos los individuos parásitos independiente de su identidad taxonómica en un individuo hospedador, la diversidad y la dominancia, que fueron calculadas con el índice de Brillouin (Magurran 1988). Aunque Simberloff \& Moore (1997) y otros autores (Poulin 1998) han conside- rado que los índices de diversidad adolecen de numerosos defectos (en lo que coincidimos), en este estudio los consideramos sólo como meros descriptores sintéticos de propiedades agregadas de las comunidades (ver Micheli et al. 1999).

La composición de las infracomunidades fue evaluada con un vector de abundancias relativas expresadas como porcentajes del total de individuos parásitos de cada taxon en la infracomunidad. Luego, se realizó un análisis de correspondencia con efecto de arco corregido (DCA, Detrended Correspondence Analysis) que permitió ordenar las infracomunidades en un espacio reducido a una dimensión. La composición de las infracomunidades se representa a través de los puntajes de los taxa en el primer eje de ordenación cuyas unidades son desviaciones estándar ( $\mathrm{x}$ 100) de la tasa de reemplazo de taxa a lo largo del gradiente.

Para realizar el DCA se consideró sólo a los taxa que tenían al menos 10 ocurrencias en las 86 infracomunidades $(\mathrm{n}=13)$, debido a la sensibilidad del análisis a las especies raras y escasas (Jongman et al. 1995). La persistencia entre años 
de muestreo de las relaciones entre la composición, riqueza, diversidad, abundancia y dominancia con la ontogenia del hospedador, fueron evaluadas a través de análisis de covarianza en que la covariable era la longitud total del huésped y el factor era el año de muestreo. Cuando no se detectaron diferencias significativas en las pendientes, se compararon las medias ajustadas (Sokal \& Rohlf 1981).

\section{RESULTADOS}

En ambos años fueron recolectados 26 taxa, de los cuales 10 eran endoparásitos larvales, nueve endoparásitos adultos y siete ectoparásitos. El 69 $\%$ de los 26 taxa ocurrieron en ambos años (Tabla $1)$.

La prevalencia de los taxa estuvo positivamente correlacionada entre años $(\mathrm{r}=0,52, \mathrm{n}=26, \mathrm{P}=$ $0,005)$, pero no la abundancia $(r=0,37, P=0,09$,
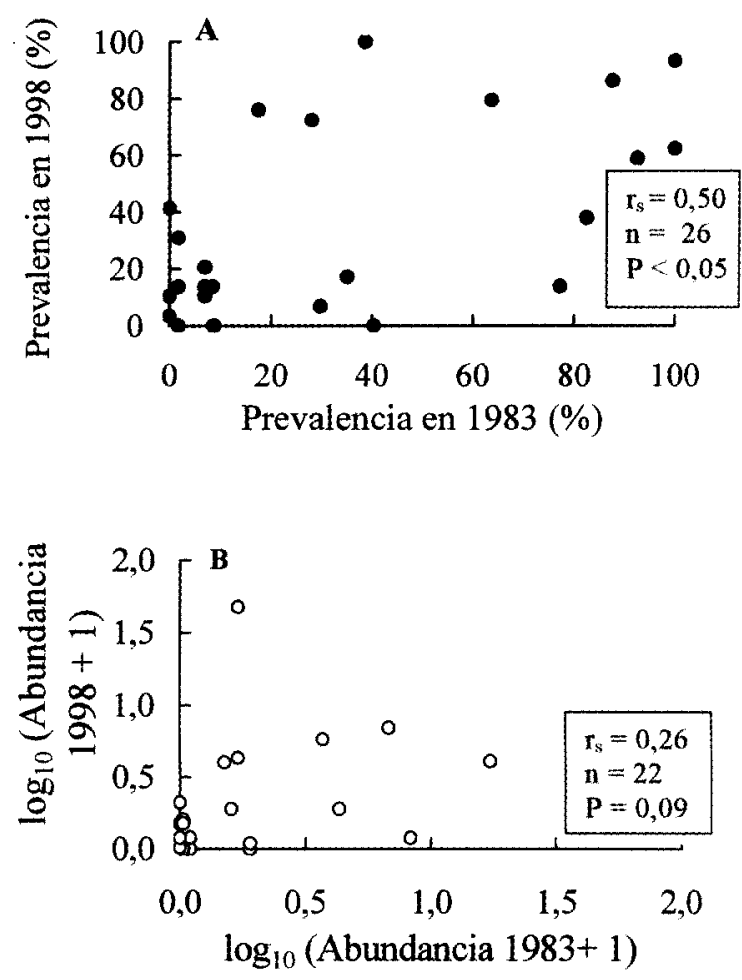

Fig. 1: Relación entre años de la (A) prevalencia $(\mathrm{n}=26)$, y $(\mathrm{B})$ abundancia $(\mathrm{n}=22)$ de los parásitos metazoos de la corvina. Se indica el coeficiente de correlación de Spearman $\left(r_{s}\right)$ y el valor de probabilidad $(\mathrm{P})$.

Between-years relationship of $(A)$ prevalence $(n=26)$ and (B) abundance $(\mathrm{n}=22)$ of metazoan parasites of the croacker. Spearman correlation coefficient $(r)$ and probability value $(\mathrm{P})$ are indicated. $\mathrm{n}=22$; Tabla 1, Fig. 1). La composición de las infracomunidades está representada por la correspondencia en el primer eje del DCA de los taxa parasitarios y de las infracomunidades, la que mostró un valor propio (autovalor) de 0,54 (Fig. 2). Allí se observa que en 1983 el taxon relativamente más abundante en las infracomunidades fue el cistacanto del acantocéfalo Corynosoma sp., y el digeneo Villarrealina peruanus en 1998 (Tabla 1, Fig. 2). Por ello, la composición de las infracomunidades resultó ser significativamente diferente entre años (Tabla 1, 2, Fig. 2).

Las variaciones de la diversidad, abundancia total y dominancia entre años se deben a las corvinas de longitud corporal inferior a $41 \mathrm{~cm}$, ya que al considerar sólo a corvinas de similar longitud total (mayores a $41 \mathrm{~cm}, \mathrm{n}=77$ ), se observaron diferencias significativas entre años sólo en composición (Tabla 2, Fig. 2 y 3 ).

\section{DISCUSIÓN}

La búsqueda de patrones en ecología, y en ecología de parásitos específicamente, ha sido considerada inútil, ante tanta multicausalidad (Poulin 1997, Simberloff \& Moore 1997). Sin embargo, es prematuro renunciar a la caracterización de la variabilidad temporal de las comunidades. La mayoría de los estudios en la variabilidad temporal de la biodiversidad (sensu Hubbell 2001), raramente considera ambas dimensiones comunitarias (composicional y agregada, sensu Micheli et al. 1999). Muchos de los estudios que dicen que tratan de comunidades de parásitos, además, describen sólo las variaciones de la prevalencia y/o abundancia de cada taxon, y no de la composición conjunta con las propiedades agregadas como la diversidad, equitabilidad, riqueza de especies, etc. (e.g., Oliva \& Luque 1998).

En la corvina, los taxa más frecuentes tendían a ser los mismos en ambos años (Tabla 1, Fig. 1). Además, en general, los parásitos de mayor prevalencia poseen mayor intensidad, y por ende, mayor abundancia (George-Nascimento 1991). Por esto, es lamentable que no se contara con los datos de abundancia de Bucephalidae gen. sp., Plagioporus sp., Cryptogonimidae gen. sp. y Philometridae gen. sp. en 1983, ya que dos de estos taxa fueron de los más numerosos en 1998. Cabe esperar que en la mayoría de las especies de hospedadores ocurra que no se alcance un valor significativo en estas correlaciones porque el número de datos depende de la riqueza de la comunidad componente, que usualmente es menor que en la corvina. 

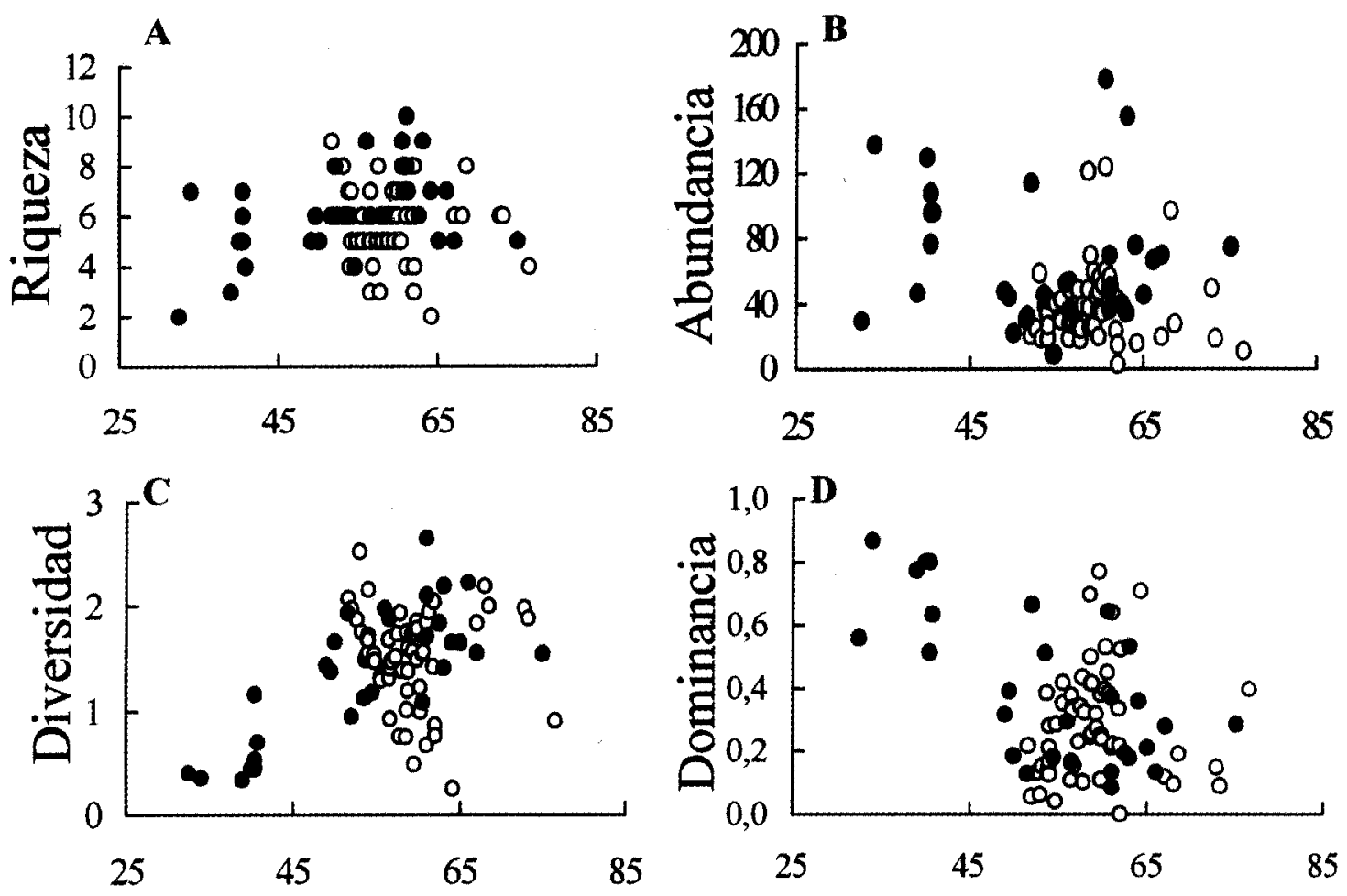

Longitud corporal $(\mathrm{cm})$

Fig. 2: Relación de la: (A) riqueza, (B) abundancia total, (C) diversidad y (D) dominancia, con la longitud total (cm) de 57 Cilus gilberti muestreadas en 1983 (símbolos vacíos) y 29 muestreadas en 1998 (símbolos negros).

Relationship of (A) richness, (B) total abundance, (C) diversity and (D) dominance, with total body length (cm) of 57 Cilus gilberti sampled in 1983 (open symbols), and 29 sampled in 1998 (filled symbols).

La corvina está entre las especies con mayor riqueza de parásitos a nivel infracomunitario y componente, entre las cerca de 50 especies de peces marinos que han sido estudiadas por parásitos en la costa de Chile (M. George-Nascimento resultados no publicados). Por esto, es en esta especie de huésped donde es más probable que las interacciones entre algunas de las especies de parásitos puedan al menos ocurrir. En la corvina, precisamente éstas podrían darse entre los digeneos del tracto digestivo.

¿Qué podría ayudar a explicar que entre años sólo la composición varíe significativamente, y por el contrario, que las propiedades agregadas sean más persistentes en las infracomunidades?. Si la variación ontogenética del uso del hábitat y de la dieta tiende a mantenerse entre años dentro de cada especie de hospedador, como por ejemplo en Aplodactylus punctatus (Benavides et. al. 1994), se podría esperar una estabilidad entre años de la relación de la composición y de los descriptores infracomunitarios con la edad del hospedador. Por contraposición, los resultados muestran que las variaciones en composición de las infracomunidades de parásitos de la corvina se deben a las diferencias entre años en la abundancia relativa de sólo algunos taxa, principalmente transmitidos vía trófica (Corynosoma y $V$. peruanus, Fig. 3). Esto sugiere que variaciones entre años en el consumo de las presas podrían haber afectado la importancia relativa de los tipos de presa en cada nivel ontogenético, provocando variaciones entre años de la abundancia relativa de cada taxón parasitario, y por tanto en la composición infracomunitaria.

Al parecer, es general el que hay poca variación entre años en la composición, y en las propiedades agregadas de las infracomunidades de parásitos de los peces marinos ya que estos resultados, los de Balboa \& George-Nascimento (1998), y los de otras tres especies de peces marinos muestran resultados similares (resultados no publicados, $M$ George-Nascimento). Si esta baja variabilidad composicional y agregada es representativa del patrón de los parásitos de peces, estas comunidades yacerían en el sector en que predomina la 


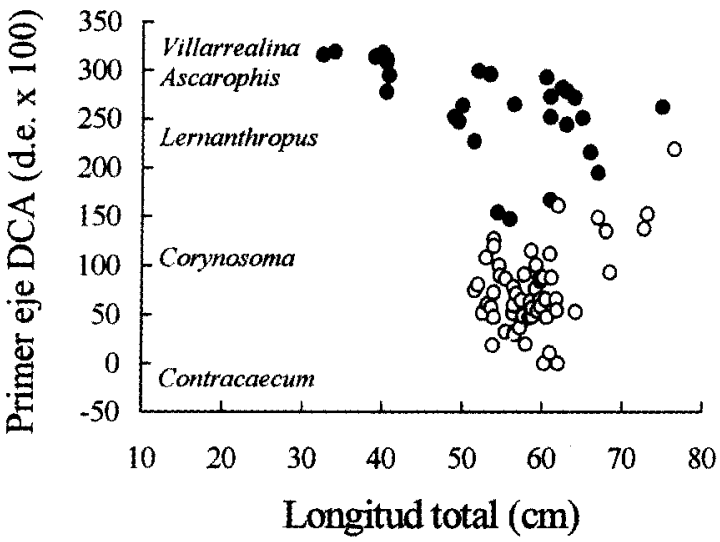

Fig. 3: Relación entre el primer gradiente de composición de la fauna parasitaria de la corvina Cilus gilberti (expresado en unidades de desviación estándar x 100), y la longitud total de los hospedadores $(\mathrm{cm})$, según el año 1983 (símbolos vacíos) y el año 1998 (símbolos negros). Los nombres de los taxa parasitarios se indican frente a su posición en el primer eje del DCA.

Relationship between the first DCA compositional gradient of the parasitic fauna of the croaker Cilus gilberti (expressed in standard deviation units $x 100$ ), and total body length of the host $(\mathrm{cm})$, according to the year of sampling: 1983 (open symbols) 1998 (filled symbols). Names of parasite taxa are shown in front of their position on the first DCA axis.

estasis o la compensación comunitaria (ver Micheli et al. 1999). Aunque estos patrones no permiten deducir qué factor(es) lo(s) origina(n), es dudoso que en las infracomunidades de parásitos de Helicolenus lengerichi y Sebastes capensis las interacciones biológicas entre los parásitos sean una explicación adecuada para este patrón, ya que no habría un escenario propicio para ellas (ver George-Nascimento \& Iriarte 1989, Balboa \& George-Nascimento 1998).

\section{AGRADECIMIENTOS}

Este trabajo fue financiado por el proyecto FONDECYT 1980079 y por el Proyecto FONDAP, programa 3, Oceanografía y Biología Marina, Ecología y Conservación.

\section{LITERATURA CITADA}

BALBOA L \& M GEORGE-NASCIMENTO (1998) Variaciones ontogenéticas y entre años en las infracomunidades de parásitos metazoos de dos especies de peces marinos de Chile. Revista Chilena de Historia Natural 71: 27-37.
BENAVIDES AG, JM CANCINO \& FP OJEDA (1994) Ontogenetic changes in gut dimensions and macroalgal digestibility in the marine hebivorous fish, Aplodactylus punctatus. Functional Ecology 8: 4651.

BOLAÑOS LL \& EM SALAS (1982) Villarrealina peruanus gen. et sp. n. (Digenea: Allocreadiidae) en el pez marino Sciaena gilberti. Investigación y Ciencia, Universidad Federico Villarreal (Perú) 1: 51-53.

CANNON LR (1977) Some larval ascaridoids from southeastern Queensland marine fishes. International Journal for Parasitology 7: 233-243.

CHONG J, N CORTÉS \& R BUSTOS (2000) Hábitos alimentarios de la corvina Cilus gilberti (Abbott 1889) (Pisces: Sciaenidae) frente a la costa de Talcahuano. Biología Pesquera (Chile) 28: 29-35.

DEARDORFF TL \& RM OVERSTREET (1981) Larval Hysterothylacium sp. (= Thynnascaris) (Nematoda: Anisakidae) from fishes and invertebrates in the Gulf of México. Proceedings of the Helminthological Society of Washington 48: 113-126.

ESCH GW, A BUSH \& J AHO (1990a) Parasite communities: patterns and processes. Chapman and Hall, London, United Kingdom. 335 pp.

ESCH WP, AW SHOSTAK, DJ MARCOGLIESE \& TM GOATER (1990b) Patterns and processes in helminth parasite communities: an overview. En: Esch GW, AO Bush \& JM Aho (eds) Parasite communities: patterns and processes: 1-19. Chapman-Hall, New York, New York.

GEORGE-NASCIMENTO M (1991) La estructura de los ensambles comunitarios de helmintos parásitos de vertebrados marinos: un acercamiento a distintos niveles jerárquicos. Tesis Doctoral, Facultad de Ciencias Biológicas, Pontificia Universidad Católica de Chile, Santiago, Chile. 332 pp.

GEORGE-NASCIMENTO M \& JL IRIARTE (1989) Las infracomunidades de parásitos metazoos del chancharro Helicolenus lengerichi Norman 1937 (Pisces, Scorpaenidae): un ensamble no interactivo de especies. Revista Chilena de Historia Natural 62: 217-227.

GRUNER L, M MALCZEWSKI, G JAKUB, N BOGUSLAW, K JEDRZEJ \& J BOIUX (1998) Stability of nematode parasite communities of sheep in a Polish flock in relation to years, seasons and resistance status of hosts. Acta Parasitologica 43: 154-161

HOLMES JC (1990) Helminth communities in marine fishes. En: Esch GW, AO Bush \& JM Aho (eds) Parasite communities: patterns and processes: 101130. Chapman-Hall, New York, New York.

HOLMES JC \& PW PRICE (1986) Communities of parasites. En: Anderson DJ \& J Kikkawa (eds) Community ecology: pattern and processes: 187-213. Blackwell Scientific Publications, Oxford, United Kingdom.

HUBBELL SP (2001) The unified neutral theory of biodiversity and biogeography. Princeton University Press, Princeton, New Jersey. 390 pp.

JAKSIC F (2001) Ecología de comunidades. Ediciones Universidad Católica de Chile, Santiago, Chile. 233 pp. 
JONGMAN RHG, CJF TER BRAAK \& OFR VAN TONGEREN (1995) Data analysis in community and landscape ecology. Cambridge University Press, New York, New York. 299 pp.

KENNEDY CR (1993) The dynamics of intestinal helminth communities in eels Anguilla anguilla in a small stream: long-term changes in richness and structure. Parasitology 107: 71-78.

KONG I \& J VALDÉS (1990) Sciaenidos de Chile: análisis taxonómico y morfológico. Estudios Oceanológicos (Chile) 9: 13-56.

MAGURRAN AE (1988) Ecological diversity and its measurement. Princeton University Press, Princeton, New Jersey. $175 \mathrm{pp}$.

MARGOLIS L, GW ESCH, JC HOLMES, AM KURIS \& GA SCHAD (1982) The use of ecological terms in parasitology. Journal of Parasitology 68: 131-133.

McGOWAN JA \& PW WALKER (1993) Pelagic diversity patterns. En: Ricklefs RE \& D Schluter (eds) Species diversity in ecological communities. Historical and geographical perspectives: 203-214. The University of Chicago Press, Chicago, Illinois.

MENDOZA R (1983) Contribución al conocimiento del parasitismo en la corvina Cilus gilberti (Abbot, 1899) (Pisces: Teleostei). Memoria presentada para optar al título de Médico Veterinario, Universidad de Concepción, Chillán, Chile. 71 pp.

MICHELI F, KL COTTINGHAM, J BASCOMPTE, ON BJORNSTAD, GL ECKERT, JM FISCHER, TH KEITH, BE KENDALL, JL KLUG \& JA RUSAK (1999) The dual nature of community variability. Oikos 85: 161-169.

OLIVA M \& L DURÁN (1982) Lernanthropus pacificus $\mathrm{n}$. sp. (Crustacea: Copepoda) parásito en la corvinilla Sciaena gilberti (Abbott) (Pisces: Teleostei). Boletín Chileno de Parasitología 37: 15-17.

OLIVA M \& JL LUQUE (1998) Metazoan parasite infracommunities in five sciaenids from the central Peruvian coast. Memorias do Instituto Oswaldo Cruz, Rio de Janeiro (Brasil) 93: 175-180.

OYARZÚN C, G HERRERA \& JR LAVENBERG (1985) Status taxonómico de la corvina Cilus montti Delfin 1990 (Pisces: Sciaenidae). Biología Pesquera (Chile) 14: $40-45$.

PIMM S (1991) The balance of nature. The University of Chicago Press, Chicago, Illinois. 448 pp.
POULIN R (1997) Species richness of parasite assemblages: evolution and patterns. Annual Review of Ecology and Systematics 28: 341-358.

POULIN R (1998) Evolutionary ecology of parasites: from individuals to communities. Chapman \& Hall, London, United Kingdom. 212 pp.

POULIN R \& S MORAND (2000) The diversity of parasites. The Quarterly Review of Biology 75: 277293.

RICKLEFS RE \& D SCHLUTER (1993) Species diversity in ecological communities. Historical and geographical perspectives. The University of Chicago Press, Chicago, Illinois. 416 pp.

SCHMIDT GD (1970) How to know the tapeworms. W.M.C. Brown Company Publishers, Dubuque, Iowa $355 \mathrm{pp}$.

SIMBERLOFF D \& J MOORE (1997) Community ecology of parasites and free-living animals. En: Clayton DH \& J Moore (eds) Host-parasite evolution: 174-197. Oxford University Press, New York, New York.

SOKAL RR \& FJ ROHLF (1981) Biometry. W. H. Freeman and Co., San Francisco, California. 859 pp.

SOUSA WP (1994) Patterns and processes in communities of helminth parasites. Trends in Ecology and Evolution 9: 52-57.

STUARDO J \& E FAGETTI (1961) Copépodos parásitos chilenos I. Una lista de las especies conocidas y descripción de 3 nuevas especies. Revista Chilena de Historia Natural 55: 55-83.

THONEY DA (1993) Community ecology of the parasites of adult spot, Leiostomus xanthurus, and Atlantic croaker, Micropogonias undulatus (Sciaenidae) in the Cape Hatteras region. Journal of Fish Biology 43 718-804.

VICKERY WL \& R POULIN (1998) Parasite extinction and colonization, and the evolution of parasite communities: a simulation study. International Journal for Parasitology 28: 727-737.

VILLALBA C \& J FERNÁNDEZ (1984) Lernanthropus guacoldae (Copepoda: Lernanthropidae), parásito de Sciaena deliciosa (Tschudi 1984) (Pisces: Sciaenidae) en Chile. Boletín de la Sociedad de Biología Concepción (Chile) 55: 127-133.

ZAR JH (1984) Biostatistical analysis. Segunda edición. Prentice-Hall, Inc., Englewood Cliffs, New Jersey. $718 \mathrm{pp}$.

Editor Asociado: P. Ojeda

Recibido el 16 de octubre de 2000; aceptado el 23 de agosto de 2001 